九州大学学術情報リポジトリ

Kyushu University Institutional Repository

\title{
On the local factor of the zeta function of quadratic orders
}

Kaneko, Masanobu

Faculty of Mathematics, Kyushu University

http://hdl. handle. net/2324/11826

出版情報: Zeta Functions, Topology and Quantum Physics, pp.75-79, 2005. Springer バージョン :

権利関係 : 


\title{
MHF Preprint Series
}

Kyushu University

21st Century COE Program

Development of Dynamic Mathematics with

High Functionality

\section{On the local factor of the zeta function of quadratic orders}

\author{
M. Kaneko
}

MHF 2003-11

( Received December 31, 2003 )

Faculty of Mathematics

Kyushu University

Fukuoka, JAPAN 


\title{
On the local factor of the zeta function of quadratic orders
}

\section{MASANOBU KANEKO}

\begin{abstract}
We prove by an elementary method the Riemann hypothesis for the local Euler factor of the zeta function of quadratic orders.
\end{abstract}

Let $K$ be a quadratic number field, $\mathcal{O}_{K}$ its ring of integers, and $\mathcal{O}_{f}$ for each integer $f \geq 1$ the order of conductor $f$ in $\mathcal{O}_{K}=\mathcal{O}_{1}$. As shown in [1] and [3], the zeta function $\zeta_{\mathcal{O}_{f}}(s)$ of $\mathcal{O}_{f}$, which is defined by

$$
\zeta_{\mathcal{O}_{f}}(s)=\sum_{\mathfrak{a}} \frac{1}{N(\mathfrak{a})^{s}}
$$

where the sum extends over all proper ideals $\mathfrak{a}$ of $\mathcal{O}_{f}$ with norm $N(\mathfrak{a})$, has the following form:

$$
\zeta_{\mathcal{O}_{f}}(s)=\zeta_{K}(s) \cdot \prod_{p \mid f} \frac{\left(1-p^{-s}\right)\left(1-\chi(p) p^{-s}\right)-p^{n_{p}-1-2 n_{p} s}\left(1-p^{1-s}\right)\left(\chi(p)-p^{1-s}\right)}{\left(1-p^{1-2 s}\right)} .
$$

Here, $\zeta_{K}(s)$ is the Dedekind zeta function of $K$, the product is over the prime factors $p$ of the conductor $f$, with $n_{p}$ being the exact power of $p$ in $f$, and $\chi$ is the Dirichlet character corresponding to the extension $K / \mathbf{Q}$.

The main purpose of this short note is to provide proofs of the following properties, including the "Riemann hypothesis," for the local factor

$$
\varepsilon_{f, p}(s):=\frac{\left(1-p^{-s}\right)\left(1-\chi(p) p^{-s}\right)-p^{n_{p}-1-2 n_{p} s}\left(1-p^{1-s}\right)\left(\chi(p)-p^{1-s}\right)}{\left(1-p^{1-2 s}\right)}
$$

of $\zeta_{\mathcal{O}_{f}}(s) / \zeta_{K}(s)$

Theorem. 1) The function $\varepsilon_{f, p}(s)$ is a polynomial of degree $2 n_{p}$ in $p^{-s}$ and satisfies the functional equation

$$
\varepsilon_{f, p}(1-s)=p^{-n_{p}(1-2 s)} \varepsilon_{f, p}(s)
$$

2) All the zeros of $\varepsilon_{f, p}(s)$ lie on the line $\operatorname{Re}(s)=1 / 2$.

Proof. Setting $u=p^{-s}$ and $n=n_{p}$, we rewrite $\varepsilon_{f, p}(s)$ as the function $P_{n}(u)$, given as follows:

$$
\begin{aligned}
P_{n}(u) & =\frac{(1-u)(1-\chi(p) u)-p^{n-1} u^{2 n}(1-p u)(\chi(p)-p u)}{1-p u^{2}} \\
& =\frac{1-p^{n+1} u^{2(n+1)}-(1+\chi(p)) u\left(1-p^{n} u^{2 n}\right)+\chi(p) u^{2}\left(1-p^{n-1} u^{2(n-1)}\right)}{1-p u^{2}} .
\end{aligned}
$$

2000 Mathematics Subject Classification: Primary 11R42; Secondary 11M38.

Key Words and Phrases: zeta function, Riemann hypothesis. 
The numerator of this expression vanishes if we set $u= \pm 1 / \sqrt{p}$ and hence is divisible by the denominator $1-p u^{2}$. Thus $P_{n}(u)$ is indeed a polynomial of degree $2 n$. By direct division, we find

$$
P_{n}(u)=1-(1+\chi(p)) u+\cdots-p^{n-1}(1+\chi(p)) u^{2 n-1}+p^{n} u^{2 n} .
$$

The functional equation can be verified straightforwardly. This ends the proof of assertion 1).

To prove the Riemann hypothesis 2$)$, put $s=1 / 2+i t / \log p$. Then we have $u=p^{-1 / 2} e^{-i t}$ and

$$
P_{n}(u)=\frac{1-e^{-2(n+1) i t}-(1+\chi(p)) p^{-1 / 2} e^{-i t}\left(1-e^{-2 n i t}\right)+\chi(p) p^{-1} e^{-2 i t}\left(1-e^{-2(n-1) i t}\right)}{1-e^{-2 i t}} .
$$

Then, using the relation

we obtain

$$
\frac{1-e^{-2 m i t}}{1-e^{-2 i t}}=\frac{e^{-m i t}}{e^{-i t}} \frac{\sin m t}{\sin t},
$$

$$
P_{n}(u)=\frac{e^{-n i t}}{p}\left(p \frac{\sin (n+1) t}{\sin t}-\sqrt{p}(1+\chi(p)) \frac{\sin n t}{\sin t}-\chi(p) \frac{\sin (n-1) t}{\sin t}\right) .
$$

We have to show that if the right-hand side of this is zero then $t$ is real. Recall that, for any integer $m \geq 0$, the quotient $\sin (m+1) t / \sin t$ is a polynomial of degree $m$ in $x=\cos t$, which is referred to as the Chebyshev polynomial of the second kind, denoted by $U_{m}(x)$. The first several of these are as follows:

$$
U_{0}(x)=1, \quad U_{1}(x)=2 x, \quad U_{2}(x)=4 x^{2}-1, \quad U_{3}(x)=8 x^{3}-4 x .
$$

Note that the function $U_{m}(x)$ can also be defined for $m<0$; in particular, we have $U_{-1}(x)=0$ and $U_{-2}(x)=-1$. Using the $U_{m}(x)$, the proof is reduced to showing that all the roots of the polynomial (of degree $n$ )

$$
Q_{n}(x):=p U_{n}(x)-\sqrt{p}(1+\chi(p)) U_{n-1}(x)+\chi(p) U_{n-2}(x) \quad(n \geq 1)
$$

are in the real interval $[-1,1]$.

Because of the recurrence of the Chebyshev polynomials

$$
U_{n}(x)=2 x U_{n-1}(x)-U_{n-2}(x),
$$

the polynomials $Q_{n}(x)$ satisfy the same recurrence:

$$
Q_{n}(x)=2 x Q_{n-1}(x)-Q_{n-2}(x) \quad(n \geq 2),
$$

with $Q_{0}(x)=p-\chi(p)$. We show that the $n$ roots of $Q_{n}(x)$ are all in the interval $(-1,1)$ by making use of the theorem of Sturm (cf. [2, §92]), utilizing the fact that $Q_{n}(x), Q_{n-1}(x), \ldots, Q_{0}(x)$ forms a "Strum sequence". Because $U_{n}(1)=n+1$ and $U_{n}(-1)=(-1)^{n}(n+1)$, we have

$$
\begin{aligned}
Q_{n}(1) & =p(n+1)-\sqrt{p}(1+\chi(p)) n+\chi(p)(n-1) \\
& =(\sqrt{p}-1)(\sqrt{p}-\chi(p)) n+p-\chi(p)>0
\end{aligned}
$$

and

$$
\begin{aligned}
Q_{n}(-1) & =p(-1)^{n}(n+1)-\sqrt{p}(1+\chi(p))(-1)^{n-1} n+\chi(p)(-1)^{n-2}(n-1) \\
& =(-1)^{n}\{p(n+1)+\sqrt{p}(1+\chi(p)) n+\chi(p)(n-1)\} \\
& =(-1)^{n}\{(\sqrt{p}+1)(\sqrt{p}+\chi(p)) n+p-\chi(p)\} .
\end{aligned}
$$


The sign of the last expression is $(-1)^{n}$, and hence the number of "variations," as defined in $[2, \S 92]$, is $n$. Then, noting the theorem of Sturm, we conclude that $Q_{n}(x)$ has $n$ roots in the interval $(-1,1)$. (Note that, since the degree of $Q_{n}(x)$ is $n$, condition 4 of $[2, \S 92]$ need not be verified.)

Remarks and questions. 1) It is amusing that the properties stated in the above theorem are precisely those enjoyed by the congruence zeta function (or, rather, its essential part) of a curve of genus $n=n_{p}$ over the prime field $\mathbf{F}_{p}$. This naturally leads us to wonder if $\varepsilon_{f, p}(s)$ admits a cohomological (or any other "nice") interpretation and if the above theorem can be proved "conceptually" using such an interpretation.

2) The theorem proved here shows in particular that the quotient $\zeta_{\mathcal{O}_{f}}(s) / \zeta_{K}(s)$ is entire and that the Riemann hypothesis holds for $\zeta_{\mathcal{O}_{f}}(s)$ only if it holds for $\zeta_{K}(s)$. It is known that for a Galois extension $k^{\prime} / k$ of number fields, the quotient of the Dedekind zeta functions $\zeta_{k^{\prime}}(s) / \zeta_{k}(s)$ is entire. Thus the zeta function of the over field is divisible by that of the base field. On the contrary, in the case considered above, the zeta function of the subring $\mathcal{O}_{f}$ is divisible by that of the over ring. What is the reason for this?

3) The generating function of the polynomials $P_{n}(x)$ takes the simple form

$$
F(u, X):=1+\sum_{n=1}^{\infty} P_{n}(u) X^{n}=\frac{(1-u X)(1-\chi(p) u X)}{(1-X)\left(1-p u^{2} X\right)}
$$

and the functional equation for $P_{n}(u)$, which is written as

$$
p^{n} u^{2 n} P_{n}\left(\frac{1}{p u}\right)=P_{n}(u)
$$

is encoded as

$$
F\left(\frac{1}{p u}, p u^{2} X\right)=F(u, X)
$$

4) For another zeta function

$$
\zeta_{\mathcal{O}_{f}}^{*}(s)=\sum_{\mathfrak{a}} \frac{1}{N(\mathfrak{a})^{s}}
$$

where $\mathfrak{a}$ runs over all (not necessarily proper) ideals of $\mathcal{O}_{f}$, the Euler product is (cf. [3])

$$
\zeta_{K}(s) \cdot \prod_{p \mid f} \frac{1-p^{\left(n_{p}+1\right)(1-2 s)}-\chi(p) p^{-s}\left(1-p^{n_{p}(1-2 s)}\right)}{1-p^{1-2 s}} .
$$

It can be shown similarly that the local factor

$$
\frac{1-p^{\left(n_{p}+1\right)(1-2 s)}-\chi(p) p^{-s}\left(1-p^{n_{p}(1-2 s)}\right)}{1-p^{1-2 s}}
$$

possesses the same properties as in the theorem.

5) It would be nice to have a generalization of our theorem to the zeta functions of orders of number fields of higher degree.

Acknowledgment. The author is grateful to Christopher Deninger for his interest in the present work, without which this paper would not have been written. 


\section{References}

[1] M. Kaneko : A generalization of the Chowla-Selberg formula and the zeta functions of quadratic orders, Proc. Japan Acad., 66(A)-7 (1990), 201-203.

[2] H. Weber : Lehrbuch der Algebra, Vol. 1, Chelsea, New York.

[3] D. Zagier : Modular forms whose Fourier coefficients involve zeta functions of quadratic fields, in Modular functions of one variable VI, Lect. Notes in Math., no. 627, SpringerVerlag, (1977) 105-169.

Graduate School of Mathematics, Kyushu University 33,

Fukuoka, 812-8581 JAPAN

mkaneko@math.kyushu-u.ac.jp 


\section{List of MHF Preprint Series, Kyushu University 21st Century COE Program \\ Development of Dynamic Mathematics with High Functionality}

\section{$\mathrm{MHF}$}

2003-1 Mitsuhiro T. NAKAO, Kouji HASHIMOTO \& Yoshitaka WATANABE A numerical method to verify the invertibility of linear elliptic operators with applications to nonlinear problems

2003-2 Masahisa TABATA \& Daisuke TAGAMI

Error estimates of finite element methods for nonstationary thermal convection problems with temperature-dependent coefficients

2003-3 Tomohiro ANDO, Sadanori KONISHI \& Seiya IMOTO

Adaptive learning machines for nonlinear classification and Bayesian information criteria

2003-4 Kazuhiro YOKOYAMA

On systems of algebraic equations with parametric exponents

2003-5 Masao ISHIKAWA \& Masato WAKAYAMA

Applications of Minor Summation Formulas III, Plücker relations, Lattice paths and Pfaffian identities

2003-6 Atsushi SUZUKI \& Masahisa TABATA

Finite element matrices in congruent subdomains and their effective use for large-scale computations

2003-7 Setsuo TANIGUCHI

Stochastic oscillatory integrals - asymptotic and exact expressions for quadratic phase functions -

2003-8 Shoki MIYAMOTO \& Atsushi YOSHIKAWA

Computable sequences in the Sobolev spaces

2003-9 Toru FUJII \& Takashi YANAGAWA

Wavelet based estimate for non-linear and non-stationary auto-regressive model

2003-10 Atsushi YOSHIKAWA

Maple and wave-front tracking - an experiment

2003-11 Masanobu KANEKO

On the local factor of the zeta function of quadratic orders 